\title{
Quenching of High-Pressure Phases of Silicon Using Femtosecond Laser-driven Shock Wave
}

\author{
Masashi TSUJINO, ${ }^{1}$ Tomokazu SANO, ${ }^{1}$ Norimasa OZAKI, ${ }^{1}$ Osami SAKATA, ${ }^{2}$ Masayuki OKOSHI, ${ }^{3}$ \\ Narumi INOUE, ${ }^{3}$ Ryosuke KODAMA ${ }^{1}$ and Akio HIROSE $^{1}$ \\ ${ }^{1}$ Graduate School of Engineering, Osaka University, 2-1 Yamada-Oka, Suita, Osaka 565-0871 \\ ${ }^{2}$ Japan Synchrotron Radiation Research Institute / SPring-8, 1-1-1 Kouto, Sayo, Sayo-gun, Hyogo 679-5198 \\ ${ }^{3}$ National Defense Academy of Japan, 1-10-20 Hashirimizu, Yokosuka, Kanagawa 239-8686
}

(Received February 13, 2008)

\begin{abstract}
High-pressure phases which are $\beta$-Sn, Imma and simple hexagonal structures of silicon are quenched using an intense femtosecond laser-driven shock wave. These high-pressure phases have never synthesized in ambient pressure by hydrostatic and conventional shock compression methods. Femtosecond laser was irradiated to single crystal-silicon with no-dopant. We confirmed the existence of these high pressure phases by analyzing the crystalline structure of the femtosecond laser irradiated silicon using grazing incidence synchrotron x-ray diffraction.
\end{abstract}

Key Words: Femtosecond laser, Silicon, Shock wave, High-pressure phase, Quenching.

\section{Introduction}

Crystalline structure of silicon is diamond under atmospheric pressure and room temperature. Increasing the pressure, the diamond structure of silicon transforms to $\beta$-Sn structure at around $10 \mathrm{GPa}$, orthorhombic structure (space group Imma) at around $13 \mathrm{GPa}$ and simple hexagonal (SH) structure at around $16 \mathrm{GPa}$. Pressure-Temperature phase diagram ${ }^{1)}$ of silicon is shown in Fig. 1. These high-pressure phases have never been quenched after pressure release using both the hydrostatic and the conventional shock compression methods. When the pressure is released from the $\beta$-Sn structure under the hydrostatic compression, the $\beta$-Sn structure of silicon transforms to the body centered cubic structure, which is called BC8 or R8. ${ }^{2,3)}$

When a femtosecond laser pulse is irradiated to silicon, the photon energy is transferred to the thermal and the kinetic energy. ${ }^{4,5)}$ The thermal energy drives the heat wave. Owing to the kinetic energy, the explosive ablation occurs. ${ }^{5)}$ The recoil force during the ablation drives the strong mechanical shock wave. Then the shock wave front overtakes and precedes the heat wave front ${ }^{6}$ and the region affected by only shock wave is formed. When the shock wave is driven inside materials, the temperature rises because of pressure rising. The temperature calculation is performed in order to confirm that silicon does not melt in spite of the temperature rising in the shock compression. We use the thermodynamic equation ${ }^{7)}$ derived by Walsh and Christian as follows:

$$
C_{V} \frac{d T}{d V}+\frac{\gamma_{0}}{V_{0}} C_{V} T=\frac{1}{2} \frac{d p}{d V}\left(V_{0}-V\right)+\frac{1}{2}\left(p-p_{0}\right),
$$

where $C_{\mathrm{V}}$ is the constant-volume specific heat, $T$ is the temperature, $V$ is the constant volume, $\gamma$ is the Grüneisen parameter, and $p$ is the pressure. Grüneisen parameter is referred from the data of Gauster. ${ }^{8)}$ The constant-volume specific heat was calculated using the equation, $C_{\mathrm{V}}=3 N k_{\mathrm{B}}$, where $N$ is the number of atoms per unit mass, $k_{\mathrm{B}}$ is the
Boltzmann constant. The calculated temperature is shown as a dashed curve in Fig. 1. The dashed curve means the maximum temperature which rises due to the shock compression. This calculation shows that silicon does not melt under shock compression.

The heat-affected zone by a femtosecond laser is smaller than another long pulse laser such as a nanosecond or a picosecond laser. Therefore, the surrounding area of the shock-affected zone rapidly absorbs the heat which rises due to the shock compression and the shock-affected zone is rapidly cooled. Because of this effect, the non-equilibrium phases are easily quenched in comparison with the conventional shock compression methods. ${ }^{9)}$

The purpose of this study is to quench the high-pressure

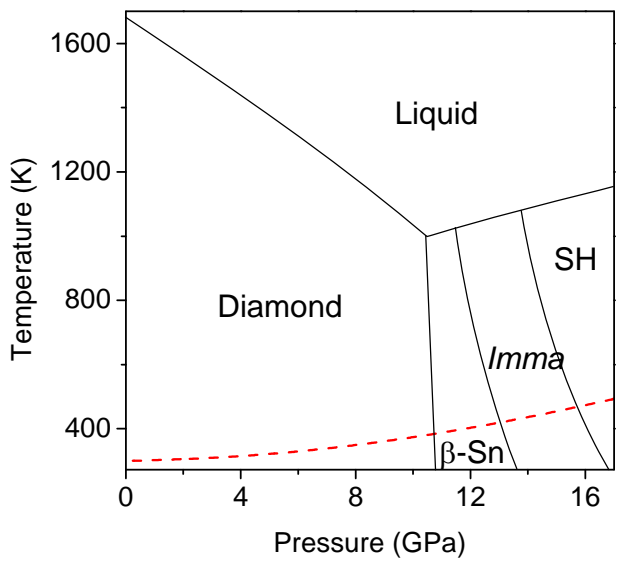

Fig. 1 Phase diagram of silicon. ${ }^{1)} \mathrm{SH}$ : simple hexagonal, dashed curve: Pressure-Temperature curve under shock compression. 
phases of silicon after pressure release using femtosecond laser-driven shock wave.

\section{Experiments}

A femtosecond laser (Spitfire, Spectra-Physics Inc.) pulse was irradiated to silicon, which was single crystal with no-dopant and had the mirror-polished surface of (100) lattice plane, in air at atmospheric pressure and room temperature. The spatial distribution of the laser energy is near-Gaussian profile. The wavelength was $800 \mathrm{~nm}$, the pulse width was $130 \mathrm{fs}$ and the pulse energy was $6.1 \mathrm{~mJ}$. The intensity, which is calculated from the irradiated spot size and effective energy, is $3 \times 10^{15} \mathrm{~W} / \mathrm{cm}^{2}$. Although this intense femtosecond laser pulse had a prepulse which might have intensity around $10^{9}-10^{10} \mathrm{~W} / \mathrm{cm}^{2}$, the intensity of a prepulse was much lower than main pulse. Therefore, the heat and shock effects by a prepulse are negligible. The observation of the surface of the femtosecond laser irradiated silicon was performed using a scanning electron microscope (SEM). In order to analyze the compound of silicon, x-ray photoelectron spectroscopy (XPS) was performed after the femtosecond laser was irradiated.

For the determination of the crystalline structures of silicon after the femtosecond laser irradiation, we used transmission electron microscopy (TEM) and performed grazing incidence $\mathrm{x}$-ray diffraction (GI-XRD) ${ }^{10)}$ measurement using synchrotron $\mathrm{x}$-ray radiation. The specimen of TEM observation was vertically cut out from the femtosecond laser irradiated spot using focused ion beam (FIB). The cross section of the irradiated spot was observed in TEM. GI-XRD was performed at a beamline for surface and interface structures, BL13XU of
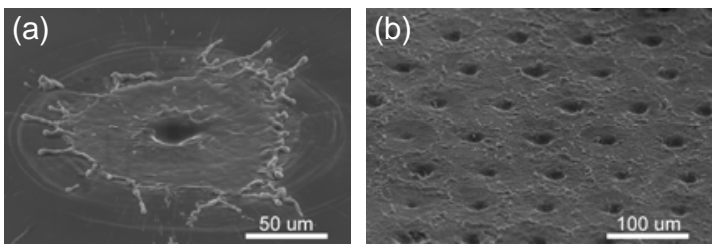

Fig. 2 SEM image of specimen surface after femtosecond laser irradiation. (a) One pulse irradiated surface. (b) The specimen for GI-XRD analyses.

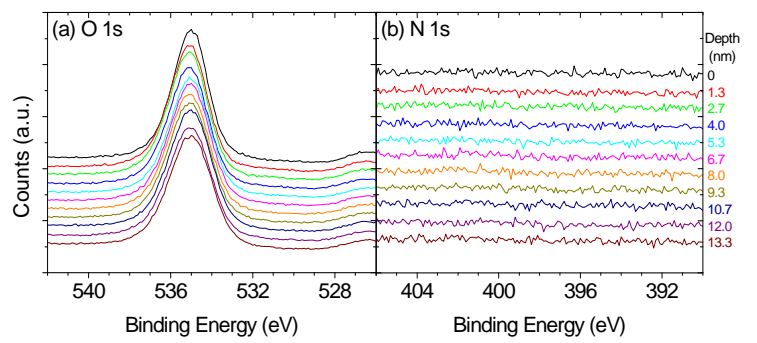

Fig. 3 XPS patterns for femtosecond laser irradiated silicon. Analyses are performed for (a) oxygen 1s orbit $\left(\mathrm{SiO}_{2}\right)$ and (b) nitrogen $1 \mathrm{~s}$ orbit $\left(\mathrm{Si}_{3} \mathrm{~N}_{4}\right)$. The upperest patterns are the analyses for the specimen surface and the lower patterns are for the deeper region of the specimen.
SPring-8. ${ }^{11)}$ In GI-XRD, the incident angle $\alpha$ was fixed and the detected angle $\delta$ was varied. Therefore, the penetration depth of the $\mathrm{x}$-ray was able to be controlled and we could analyze only the surface structure with small incident angle. The wavelength of the x-ray was $1.000 \AA$.

\section{Results and Discussion}

SEM images of femtosecond laser irradiated silicon are shown in Fig. 2. Figure 2(a) shows the single pulse irradiated surface. A crater with the depth of few microns was formed at the center of the laser irradiated spot. The injection pulse energy to the crater was $1.5 \mathrm{~mJ}$ and the intensity was estimated to be $3 \times 10^{15} \mathrm{~W} / \mathrm{cm}^{2}$ assuming that the spatial distribution of the laser energy is near-Gaussian profile. A TEM sample was cut out vertically from the sample of Fig. 2(a). The sample for the GI-XRD is shown in Fig. 2(b). A femtosecond laser pulse was irradiated to one spot and the interval between spots was $80 \mu \mathrm{m}$.

XPS patterns are shown in Fig. 3. In order to investigate the existence of the silicon compound for the depth direction, the specimen was etched using $\mathrm{Ar}^{+}$ion. The top patterns are the analyses for the specimen surface and the lower patterns are for the deeper region of the specimen. $\mathrm{SiO}_{2}$ and $\mathrm{Si}_{3} \mathrm{~N}_{4}$ might be generated, because of oxygen and nitrogen in air. XPS analyses were performed for oxygen 1s orbit $\left(\mathrm{SiO}_{2}\right)$ and nitrogen $1 \mathrm{~s}$ orbit $\left(\mathrm{Si}_{3} \mathrm{~N}_{4}\right)$. As a result of the XPS analyses, $\mathrm{SiO}_{2}$ existed and $\mathrm{Si}_{3} \mathrm{~N}_{4}$ did not exist in the femtosecond laser irradiated silicon.

A TEM image is shown in Fig. 4. The upside of the figure is the surface of the femtosecond laser irradiated silicon, and the downside is the inside of the silicon. Many defects are confirmed in the region with a depth of several microns from the surface. Therefore, the plastic deformation occurred in this region and the pressure was over the plastic deformation threshold of around $9 \mathrm{GPa}^{12)}$ Under this region, the number of

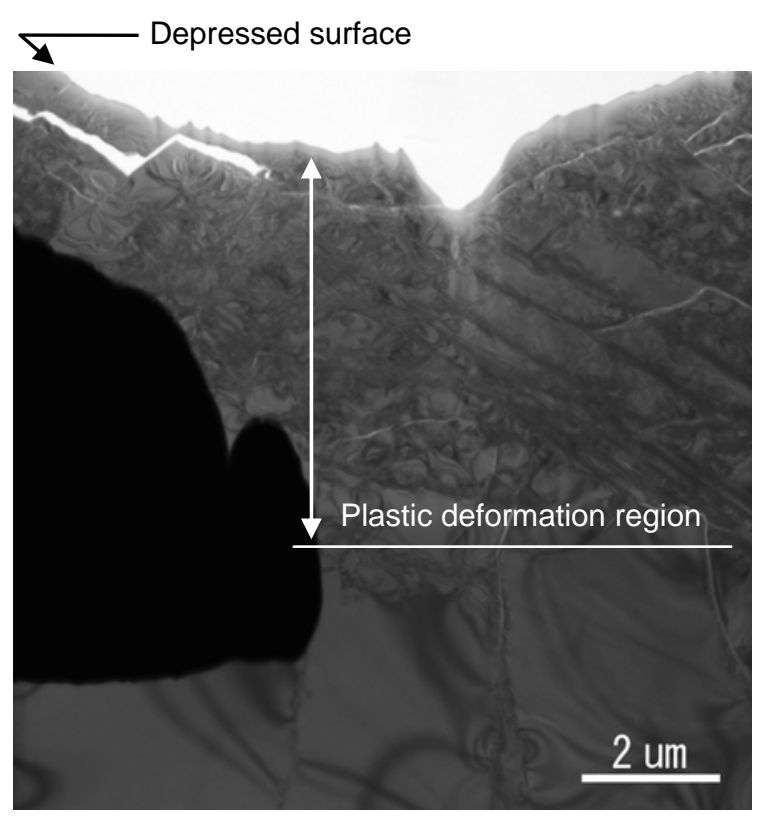

Fig. 4 TEM images of cross section of femtosecond laser irradiated spot. 
Table 1 Assignment of peaks observed from GI-XRD and comparison between observed $d$-spacings and actual $d$-spacings of high-pressure phases of silicon ${ }^{13)}$.

\begin{tabular}{cccccc}
\hline Angle (deg) & $d_{\text {obs }}(\AA)$ & Phase & hkl & $d(\AA)$ & Difference (\%) \\
\hline 24.24 & 2.381 & SH & 001 & 2.381 & 0.01 \\
24.32 & 2.375 & Imma & 200 & 2.374 & 0.03 \\
24.70 & 2.339 & $\beta-S n$ & 200 & 2.334 & 0.20 \\
25.56 & 2.261 & Imma & 020 & 2.251 & 0.46 \\
25.76 & 2.243 & -Sn & 101 & 2.242 & 0.04 \\
25.94 & 2.229 & Imma & 011 & 2.220 & 0.39 \\
26.30 & 2.198 & SH & 100 & 2.204 & -0.27 \\
\hline
\end{tabular}

the defects suddenly decreased. It is thought that the shock wave rapidly decayed. But it is suggested that the pressure must be over the phase transition threshold of around $10 \mathrm{GPa}^{1,12)}$ and the pressure for synthesizing the high-pressure phases of silicon is achieved near the specimen surface.

GI-XRD patterns are shown in Fig. 5. In the measurements of Fig. 5(a) for the wide range, $\mathrm{X}$-ray exposure time was 1 s/step. The diffractions from the lattice planes of diamond structure, which were different from the matrix plane, were detected. The tridymite- $\mathrm{SiO}_{2}$ structure which was the high-temperature phase of $\mathrm{SiO}_{2}$ was also detected. The diffraction patterns of these phases are obtained from the surface heat affected zone by laser heating. In the measurements of Fig. 5(b) for the short range, the x-ray exposure time was $5 \mathrm{~s} / \mathrm{step}$, in order to perform the more detailed analyses. Some diffraction patterns were obtained from the femtosecond laser irradiated specimen. We calculated the $d$-spacings from the angle of the obtained peaks, and those $d$-spacings were compared with ones of high-pressure phases of silicon. ${ }^{13)}$ As a result, we confirmed the existence of high-pressure phases of silicon. Those are $\beta$-tin, Imma, and SH structures. The diffractions at 24.70 deg and 25.76 deg are near ones of the $\mathrm{SiO}_{2}$ phases, but closer ones of the $\beta$-Sn phase than ones of the $\mathrm{SiO}_{2}$ phase. The other observed diffractions do not accord with the possible $\mathrm{SiO}_{2}$ polymorphs (Low quartz as stable phase, high quartz, tridymite and cristobalite as high-temperature phase, and coesite, stishovite and $\mathrm{CaCl}_{2}$-type as high-pressure phase). A list of the diffracted angle and the $d$-spacings is shown in Table 1 . Small differences between the observed and the actual $d$-spacings are caused by the strain of the crystalline structures. From this result, it was realized that the quenching of high-pressure phases of silicon is achieved using the femtosecond laser-driven shock wave.

From the TEM observation, we suggest that strong shock compression is loaded under the deep crater of the center of the irradiated spot. Diffraction peaks of high-pressure phases of silicon are considerably lower than those of the diamond phase in GI-XRD. These results may mean that the high-pressure phases only exist just under the crater formed by the laser irradiation and the region of the phase created by one pulse is extremely small.

In these experiments, the laser intensity was $3 \times 10^{15} \mathrm{~W} / \mathrm{cm}^{2}$. McGrane et al. reported that the pressure loaded by roughly same intensity was over $100 \mathrm{GPa}^{14)}$ However in this past study, the glass substrate was put on the target specimen in order to confine the ablation pressure. Because we irradiated the femtosecond laser in air and the ablation pressure was not confined, the pressure was much lower than the McGrane et
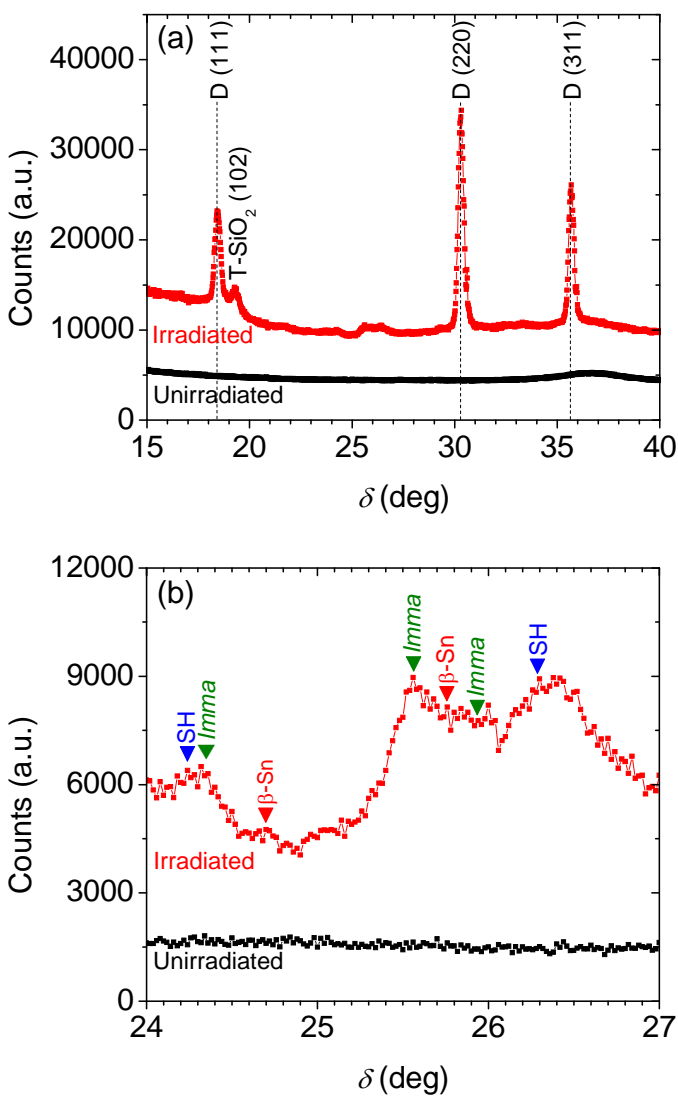

Fig. 5 Grazing incidence $\mathrm{x}$-ray diffraction patterns for femtosecond laser irradiated and unirradiated silicon. Wavelength of x-ray is $1.000 \AA$. (a) X-ray exposure time is $1 \mathrm{~s} / \mathrm{step}$. $\mathrm{D}$ : Diamond, $\mathrm{T}-\mathrm{SiO}_{2}$ : Tridymite- $\mathrm{SiO}_{2}$. (b) $\mathrm{X}$-ray exposure time is 5 s/step. SH: simple hexagonal. 
al.'s one. ${ }^{14)}$ Therefore, the pressure, which was achieved in our experiment, may be several tens GPa, and this pressure agrees with the pressure range of the quenched phase.

\section{Conclusion}

Femtosecond laser was irradiated to silicon. TEM observation and GI-XRD were performed in order to observe and analyze the crystalline structure of the femtosecond laser irradiated silicon. As a result of TEM observation, we observed many defects inside the specimen. Therefore, it was confirmed that the plastic deformation occurred and the pressure was over around $9 \mathrm{GPa}{ }^{12)}$ which was the plastic deformation threshold in this region. Near the surface, the pressure must be over the phase transition pressure around $10 \mathrm{GPa}^{12)}$ As a result of GI-XRD analyses, we confirmed the existence of the high-pressure phase of $\beta$-Sn, Imma and $\mathrm{SH}$ structures, which had never been quenched in the case of the hydrostatic and conventional shock compression methods.

\section{Acknowledgements}

This study was supported in part by "Priority Assistance for the Formation of Worldwide Renowned Centers of Research The Global COE Program (Project: Center of Excellence for Advanced Structural and Functional Materials Design)," Grant-in-Aid for Scientific Research (B), No. 17360341, and Grant-in-Aid for Scientific Research on Priority Areas "Materials Science of Bulk Metallic Glasses" from the Ministry of Education, Culture, Sports, Science and
Technology of Japan. The synchrotron radiation experiments were performed at the beamline BL13XU of SPring- 8 with the approval of the Japan Synchrotron Radiation Research Institute (Proposal Nos. 2006B1553, 2007A1638, and 2007B1681). The authors thank JEOL Ltd. for the support of TEM observation.

\section{References}

1) G. A. Voronin, C. Pantea, T. W. Zerda, L. Wang and Y. Zhao: Phys. Rev. B 68 (2003) 020102.

2) J. Crain, R. O. Piltz, G. J. Ackland, S. J. Clark, M. C. Payne, V. Milman, J. S. Lin, P. D. Hatton and Y. H. Nam: Phys. Rev. B 50 (1994) 8389.

3) R. O. Piltz, J. R. Maclean, S. J. Clark, G. J. Ackland, P. D. Hatton and J. Crain: Phys. Rev. B 52 (1995) 4072.

4) A. Ng, P. Celliers, G. Xu and A. Forsman: Phys. Rev. E 52 (1995) 4299.

5) S. K. Sundaram and E. Mazur: Nat. Mater. 1 (2002) 217.

6) A. Ng, A. Forsman and P. Celliers: Phys. Rev. E 51 (1995) R5208.

7) J. M. Walsh and R. H. Christian: Phys. Rev. 97 (1955) 1544.

8) W. B. Gauster: Phys. Rev. B 4 (1971) 1288.

9) T. Sano, H. Mori, E. Ohmura and I. Miyamoto: Appl. Phys. Lett. 83 (2003) 3498.

10) H. Dosch and B. W. Batterman: Phys. Rev. Lett. 56 (1986) 1144.

11) O. Sakata, Y. Furukawa, S. Goto, T. Mochizuki, T. Uruga, K. Takeshita, H. Ohashi, T. Ohata, T. Matsushita, S. Takahashi, et al: Surf. Rev. Lett. 10 (2003) 543.

12) W. H. Gust and E. B. Royce: J. Appl. Phys. 42 (1971) 1897.

13) M. I. McMahon and R. J. Nelmes: Phys. Rev. B 47 (1993) 8337.

14) S. D. McGrane, D. S. Moore, D. J. Funk and R. L. Rabie: Appl. Phys. Lett. 80 (2002) 3919. 